\title{
EIGENFREQUENCIES OF CURVED EULER-BERNOULLI BEAM STRUCTURES WITH DISSIPATIVE JOINTS
}

\author{
BY \\ WILLIAM H. PAULSEN \\ Arkansas State University, State University, Arkansas
}

\begin{abstract}
In this paper, we will compute asymptotically the eigenfrequencies for the in-plane vibrations of an Euler-Bernoulli beam system with dissipative joints, which allow the beams to be curved into an arc of a circle. This enhances the author's previous result for structures involving straight beams, given in his preprint "Eigenfrequencies of the non-collinearly coupled Euler-Bernoulli beam system with dissipative joints". Matrix techniques are used to combine asymptotic analysis with a form of the wave propagation method.
\end{abstract}

1. Introduction. In [14], a new method was introduced to find the in-plane vibrations of a general Euler-Bernoulli beam structure. For a straight beam, the beam equation is given by $m y_{t t}+E I y_{x x x x}=0$, with $0<x<L$ and $t>0$. Here $m$ denotes mass density per unit length, and $E I$ is the flexural rigidity of the beam. For each element of the structure, whether it be a bend, a length of beam, or a dissipative joint, a corresponding 20 by 20 matrix was given. These matrices were multiplied together, along with a 1 by 20 matrix and a 20 by 1 matrix, to form a single equation. The eigenfrequencies could easily be computed asymptotically using this equation, and it was shown that, if the lengths of the beams were rational, there would be a finite number of "streams" of eigenfrequencies lying asymptotically close to a vertical line.

In this paper we want to extend this result to allow beams in the shape of a circular arc. Because much of the setup of the problem was done in [13] and [14], the main new result will be to complete the 20 by 20 matrix corresponding to a curved beam. Once this matrix is computed, the eigenfrequencies of beam structures such as Figure 1 (see p. 260) could be computed easily.

2. Fundamental ideas. As in [13], we will use superscripts to number the elements of the structure. If the $j$ th element of the structure is a straight beam of length $l$, we let $y^{j}(x, t) \quad\left(0 \leq x \leq l_{j}, t \geq 0\right)$ denote the transverse displacement function of

Received July 2, 1992.

1991 Mathematics Subject Classification. Primary 73D30, 73K12, 35P20.

Key words and phrases. Asymptotics, curved beams, eigenfrequencies, Euler-Bernoulli, linear joints.

(C)1995 Brown University 


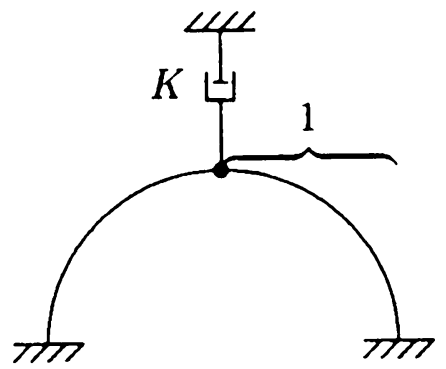

FIG. 1 .

this beam. Since this is an Euler-Bernoulli beam, we have that

$$
m \frac{\partial^{2}}{\partial t^{2}} y^{j}(x, t)+E I \frac{\partial^{4}}{\partial x^{4}} y^{j}(x, t)=0 \quad \text { for } 0<x<l_{j}, t>0 .
$$

As in [13], we will make three simplifying assumptions for this model:

(H1) The frame can vibrate only in the plane of the frame.

(H2) The beams are essentially noncompressible, that is, the change of length of the beams due to the forces exerted at the ends is negligible.

(H3) Forces exerted on a beam in the direction parallel to the length of the beam are propagated in a negligible amount of time.

Because of assumption ( $\mathrm{H} 2)$, the longitudinal displacement is independent of the position within a given beam. We let $z^{j}(t)$ denote the longitudinal displacement of the $j$ th beam. Also, because of assumption (H3), the longitudinal force of a given beam depends only on time; so we let $H^{j}(t)$ denote the longitudinal force of the $j$ th beam.

To simplify the calculations, we will use the basis of [13], given in terms of the following four functions:

$$
\begin{aligned}
& \operatorname{Hya}(x)=\frac{\cosh (x)+\cos (x)}{2}=\frac{e^{x}+e^{i x}+e^{-x}+e^{-i x}}{4}, \\
& \operatorname{Hyb}(x)=\frac{\sinh (x)-\sin (x)}{2}=\frac{e^{x}+i e^{i x}-e^{-x}-i e^{-i x}}{4}, \\
& \operatorname{Hyc}(x)=\frac{\cosh (x)-\cos (x)}{2}=\frac{e^{x}-e^{i x}+e^{-x}-e^{-i x}}{4}, \\
& \operatorname{Hyd}(x)=\frac{\sinh (x)+\sin (x)}{2}=\frac{e^{x}-i e^{i x}-e^{-x}+i e^{-i x}}{4} .
\end{aligned}
$$

We call these functions the "hybrid exponential functions". We can express the wave propagation in terms of the new functions:

$$
\begin{aligned}
y_{\lambda}^{j}(x, t)= & \left(A_{j} \operatorname{Hya}(\eta x)+B_{j} \operatorname{Hyb}(\eta x)\right. \\
& \left.+C_{j} \operatorname{Hyc}(\eta x)+D_{j} \operatorname{Hyd}(\eta x)\right) e^{\lambda t \sqrt{E I / m}}, \\
z_{\lambda}^{j}(t)= & E_{j} e^{\lambda t \sqrt{E I / m}}, \\
H_{\lambda}^{j}(t)= & F_{j} E I \eta^{3} e^{\lambda t \sqrt{E I / m}} .
\end{aligned}
$$




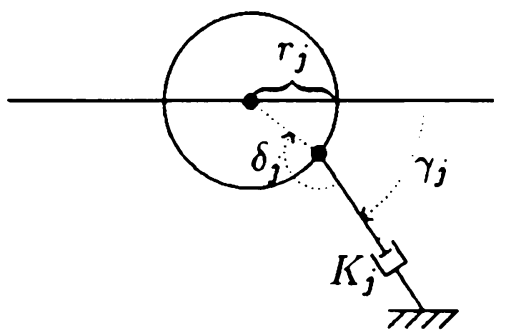

FIG. 2.

Here, $\eta=\frac{1-i}{2} \sqrt{\lambda}$, so that $i \eta^{2}=\lambda$.

The use of the hybrid exponentials deviates from the standard wave propagation method (WPM) approach to the beam equation, as described in [8]. Here, $y_{\lambda}^{j}(x, t)$ was expressed as a linear combination of four "wave functions", namely, an incoming wave $e^{(i x+\lambda t \sqrt{E I / m})}$ traveling to the right, another wave $e^{(-i x+\lambda t \sqrt{E I / m)}}$ traveling to the left, and two evanescent waves $e^{(x+\lambda t \sqrt{E I / m)}}$ and $e^{(-x+\lambda t \sqrt{E I / m})}$. The last two waves decay exponentially fast in the $x$ direction away from one of the endpoints. Thus, for each boundary condition encountered, one of the evanescent terms can safely be discarded.

In (2.2), we are expressing the wave as a sum of four "hybrid waves". Each hybrid wave is a linear combination of a wave traveling to the right, a wave traveling to the left, and the two evanescent waves. At first this may seem like a disadvantage, since all four of the hybrid waves will be important at each juncture. However, the reflection and transmission relations will be greatly simplified. For example, if the beginning end of the beam is clamped, as in the examples of [3] and [5], we can express the reflection relationships simply as $A_{j}=D_{j}=0$. That is, two of the four hybrid waves will not exist on that beam.

The price for using the hybrid wave functions is that the asymptotic estimations cannot immediately be employed by tossing out the evanescent waves. However, in the case of a curved beam, it is not completely clear that such estimations could be made anyway, since an evanescent wave may evolve into a different type of wave as it transverses the curve. Thus, we must forgo all asymptotic analysis until we have the exact equation for the eigenfrequencies. Fortunately, the asymmetrical properties of the hybrid functions allow the results to be displayed.

The boundary conditions at each joint with a damper will depend on the type of damper involved. In [14], six different types of dampers are discussed, but we will only need to understand the type III damper. Figure 2 demonstrates this type of damper. A single dashpot with a damping coefficient of $K_{j}$ is attached to the joint at a distance of $r_{j}$ from the center of the joint. The angle from the next beam to the dashpot is given by $\gamma_{j}$. If we draw a line from the point of contact of the dashpot to the center of the joint, the angle from the dashpot to this line is given by $\delta_{j}$.

The boundary conditions for this joint are computed using linear approximations to the angle displacement. For brevity, we will let $\mathbf{v}_{j}=\left(A_{j}, B_{j}, C_{j}, D_{j}, E_{j}, F_{j}\right)=$ the components of the wave in terms of the new basis after the $j$ th line in the 
description. Then the six equations can be written succinctly as

$$
\eta \mathbf{v}_{j+1}=\mathbf{v}_{j} \cdot M_{j}^{\mathrm{III}}
$$

where

$$
M_{j}^{\mathrm{III}}=\left(\begin{array}{cccccc}
\eta & -i a & i c \eta & 0 & 0 & i b \\
0 & \eta & 0 & 0 & 0 & 0 \\
0 & 0 & \eta & 0 & 0 & 0 \\
0 & -i c \eta & i f \eta^{2} & \eta & 0 & i e \eta \\
0 & -i b & i e \eta & 0 & \eta & i d \\
0 & 0 & 0 & 0 & 0 & \eta
\end{array}\right)
$$

Here,

$$
\begin{array}{lll}
a=K_{j} \sin ^{2} \gamma_{j} / \sqrt{m E I}, & b=K_{j} \cos \gamma_{j} \sin \gamma_{j} / \sqrt{m E I} \\
c=K_{j} r_{j} \sin \delta_{j} \sin \gamma_{j} / \sqrt{m E I}, & d=K_{j} \cos ^{2} \gamma_{j} / \sqrt{m E I}, \\
e=K_{j} r_{j} \sin \delta_{j} \cos \gamma_{j} / \sqrt{m E I}, & f=K_{j} r_{j}^{2} \sin ^{2} \delta_{j} / \sqrt{m E I} .
\end{array}
$$

For a general type III damper with more than one dashpot, the boundary conditions will still be the same, except that the constants $a, b, \ldots, f$ will be the sum of the corresponding constants for each dashpot.

The advantages of using the hybrid wave equations become apparent when one compares (2.4) with equations (2.12)-(2.15) of [5]. Although that paper only considers vibrations in one direction, the in-span conditions of the type III joint in terms of the standard WPM wavefunctions require almost half a page. The simplifications here, and in the other matrices, will allow us to use exponential matrix techniques later.

We call the matrix $M_{j}^{\mathrm{III}}$ the transfer matrix for the type III joint, since it indicates how the wave given by $\mathbf{v}$ propagates through the joint. The extra $\eta$ in (2.3) can be ignored, since $\eta \neq 0$.

We can also find the transfer matrices which indicate how the wave functions are transmitted across an angle, or along a straight beam of a certain length. The matrix for turning an angle $\phi$ is given by

$$
M_{j}^{\text {angle }}=\left(\begin{array}{cccccc}
\cos \phi & 0 & 0 & 0 & \sin \phi & 0 \\
0 & \cos \phi & 0 & 0 & 0 & -\sin \phi \\
0 & 0 & 1 & 0 & 0 & 0 \\
0 & 0 & 0 & 1 & 0 & 0 \\
-\sin \phi & 0 & 0 & 0 & \cos \phi & 0 \\
0 & \sin \phi & 0 & 0 & 0 & \cos \phi
\end{array}\right)
$$

The transfer matrix for a single straight beam of length $l$ is given by

$$
M_{j}^{\text {length }}=\left(\begin{array}{cccccc}
\operatorname{Hya}(l \eta) & \operatorname{Hyd}(l \eta) & \operatorname{Hyc}(l \eta) & \operatorname{Hyb}(l \eta) & 0 & 0 \\
\operatorname{Hyb}(l \eta) & \operatorname{Hya}(l \eta) & \operatorname{Hyd}(l \eta) & \operatorname{Hyc}(l \eta) & 0 & 0 \\
\operatorname{Hyc}(l \eta) & \operatorname{Hyb}(l \eta) & \operatorname{Hya}(l \eta) & \operatorname{Hyd}(l \eta) & 0 & 0 \\
\operatorname{Hyd}(l \eta) & \operatorname{Hyc}(l \eta) & \operatorname{Hyb}(l \eta) & \operatorname{Hya}(l \eta) & 0 & 0 \\
0 & 0 & 0 & 0 & 1 & 0 \\
0 & 0 & 0 & 0 & 0 & 1
\end{array}\right) .
$$


By multiplying the transfer matrices together, we find how the wave functions are propagated throughout the structure.

Finally, we need to consider the two ends of the structure. If the final end of the structure is either clamped or free, the relationships between the reflected waves can be expressed as

$$
\mathbf{v}_{n} \cdot M_{n}=\mathbf{0}
$$

where

$$
M_{n}^{\text {clamp }}=\left(\begin{array}{ccc}
1 & 0 & 0 \\
0 & 0 & 0 \\
0 & 0 & 0 \\
0 & 1 & 0 \\
0 & 0 & 1 \\
0 & 0 & 0
\end{array}\right), \quad \text { or } \quad M_{n}^{\text {free }}=\left(\begin{array}{ccc}
0 & 0 & 0 \\
1 & 0 & 0 \\
0 & 1 & 0 \\
0 & 0 & 0 \\
0 & 0 & 0 \\
0 & 0 & 1
\end{array}\right) .
$$

Other types of end boundary conditions can be obtained by combining a damper with one of the above matrices. For example, the boundary control used in [11] can be obtained by considering a type III damper immediately followed by a free end.

By combining the information from the transfer matrices, we get the equation

$$
\mathbf{v}_{1} \cdot\left(M_{1} \cdot M_{2} \cdot M_{3} \cdots M_{n}\right)=\mathbf{0} .
$$

This gives us three equations with six unknowns. To find three other equations, we consider that the vibrations of certain frequencies will induce resonance in the structure. The beginning end of the structure will be clamped, which we can express as $\mathbf{v}_{1} \cdot M_{n}^{\text {clamp }}=\mathbf{0}$. Resonance will occur if $A_{1}=D_{1}=E_{1}=0$ at the beginning of the structure. Thus, only the second, third, and sixth columns of $\left(M_{1} \cdot M_{2} \cdot M_{3} \cdots M_{n}\right)$ will be important. We can express this by letting

$$
M_{0}^{\text {clamp }}=\left(\begin{array}{cccccc}
0 & 1 & 0 & 0 & 0 & 0 \\
0 & 0 & 1 & 0 & 0 & 0 \\
0 & 0 & 0 & 0 & 0 & 1
\end{array}\right) .
$$

Then there will be a nontrivial solution to the wave equations if and only if

$$
\operatorname{det}\left(M_{0} \cdot M_{1} \cdot M_{2} \cdots M_{n}\right)=0 .
$$

Let us denote $G=M_{0} \cdot M_{1} \cdot M_{2} \cdots M_{n}$. Then whenever $\eta$ is a solution to $|G|=0$, $\lambda=i \eta^{2}$ will be an eigenfrequency of the structure.

To find the transfer matrix for a curved beam, we will divide the beam into infinitesimal beam segments. That is, we will approximate the curve with a sequence of beam structures involving straight beams, and take the limit as the lengths of the beams go to zero. For a circular arc of length $l$ and curvature $\kappa$, we can approximate the arc by a sequence of $l / \Delta l$ beams of length $\Delta l$, with an angle of $\Delta \phi=\kappa \Delta l$ between them. When $\kappa>0$, we will be turning counterclockwise as we transverse the length of the beam. Turning clockwise is indicated by choosing $\kappa<0$ with the same magnitude. It is easy to describe the matrix using the matrix exponential function, described in [2, p. 147]. We can describe the matrices for an angle and a 
beam length as

$$
\begin{gathered}
M_{j}^{\text {length }}=\exp \left(\begin{array}{cccccc}
0 & l \eta & 0 & 0 & 0 & 0 \\
0 & 0 & l \eta & 0 & 0 & 0 \\
0 & 0 & 0 & l \eta & 0 & 0 \\
l \eta & 0 & 0 & 0 & 0 & 0 \\
0 & 0 & 0 & 0 & 0 & 0 \\
0 & 0 & 0 & 0 & 0 & 0
\end{array}\right), \\
M_{j}^{\text {angle }}=\exp \left(\begin{array}{cccccc}
0 & 0 & 0 & 0 & \phi & 0 \\
0 & 0 & 0 & 0 & 0 & -\phi \\
0 & 0 & 0 & 0 & 0 & 0 \\
0 & 0 & 0 & 0 & 0 & 0 \\
-\phi & 0 & 0 & 0 & 0 & 0 \\
0 & \phi & 0 & 0 & 0 & 0
\end{array}\right) .
\end{gathered}
$$

To first order in $\Delta l$, we have that

$$
\begin{gathered}
M_{j}^{\text {length }}(\Delta l) \cdot M_{j}^{\text {angle }}(\kappa \Delta l) \\
=\exp \left[\Delta l\left(\begin{array}{cccccc}
0 & \eta & 0 & 0 & \kappa & 0 \\
0 & 0 & \eta & 0 & 0 & -\kappa \\
0 & 0 & 0 & \eta & 0 & 0 \\
\eta & 0 & 0 & 0 & 0 & 0 \\
-\kappa & 0 & 0 & 0 & 0 & 0 \\
0 & \kappa & 0 & 0 & 0 & 0
\end{array}\right)\right] .
\end{gathered}
$$

Including $l / \Delta l$ such beams amounts to raising this expression to the $l / \Delta l$ power, giving us

$$
M_{j}^{\text {curve }}=\exp \left(\begin{array}{cccccc}
0 & l \eta & 0 & 0 & l \kappa & 0 \\
0 & 0 & l \eta & 0 & 0 & -l \kappa \\
0 & 0 & 0 & l \eta & 0 & 0 \\
l \eta & 0 & 0 & 0 & 0 & 0 \\
-l \kappa & 0 & 0 & 0 & 0 & 0 \\
0 & l \kappa & 0 & 0 & 0 & 0
\end{array}\right)=\exp (A) .
$$

Before we can do any asymptotic estimates on this matrix, we need to expand the matrix exponential. Fortunately, the eigenvalues of the matrix $A$ are simple: 0,0 , $\pm l \sqrt{\eta^{2}-\kappa^{2}}$, and $\pm i l \sqrt{\eta^{2}+\kappa^{2}}$. Because of the double eigenvalue, this matrix is nondiagonalizable, but we can put this into Jordan canonical form. If we let

$$
D=\left(\begin{array}{cccccc}
0 & l \kappa & 0 & 0 & 0 & 0 \\
0 & 0 & 0 & 0 & 0 & 0 \\
0 & 0 & l \sqrt{\eta^{2}-\kappa^{2}} & 0 & 0 & 0 \\
0 & 0 & 0 & -l \sqrt{\eta^{2}-\kappa^{2}} & 0 & 0 \\
0 & 0 & 0 & 0 & i l \sqrt{\eta^{2}+\kappa^{2}} & 0 \\
0 & 0 & 0 & 0 & 0 & -i l \sqrt{\eta^{2}+\kappa^{2}}
\end{array}\right)
$$


and

$$
P=\left(\begin{array}{cccccc}
0 & 0 & \eta^{2}-\kappa^{2} & \eta^{2}-\kappa^{2} & -\eta^{2}-\kappa^{2} & -\eta^{2}-\kappa^{2} \\
0 & \eta^{2} & \eta \sqrt{\eta^{2}-\kappa^{2}} & -\eta \sqrt{\eta^{2}-\kappa^{2}} & -i \eta \sqrt{\eta^{2}+\kappa^{2}} & i \eta \sqrt{\eta^{2}+\kappa^{2}} \\
\kappa \eta & 0 & \eta^{2} & \eta^{2} & \eta^{2} & \eta^{2} \\
0 & \kappa^{2} & \eta \sqrt{\eta^{2}-\kappa^{2}} & -\eta \sqrt{\eta^{2}-\kappa^{2}} & i \eta \sqrt{\eta^{2}+\kappa^{2}} & -i \eta \sqrt{\eta^{2}+\kappa^{2}} \\
0 & -\eta^{3} / \kappa & -\kappa \sqrt{\eta^{2}-\kappa^{2}} & \kappa \sqrt{\eta^{2}-\kappa^{2}} & -i \kappa \sqrt{\eta^{2}+\kappa^{2}} & i \kappa \sqrt{\eta^{2}+\kappa^{2}} \\
\eta^{2} & 0 & \kappa \eta & \kappa \eta & -\kappa \eta & -\kappa \eta
\end{array}\right),
$$

then $A=P \cdot D \cdot P^{-1}$. We can compute $P^{-1}$ to be

$$
\frac{1}{4 \eta p q\left(\eta^{4}-\kappa^{4}\right)}\left(\begin{array}{cccccc}
-4 \kappa \eta^{2} p q & 0 & -4 \kappa^{3} p q & 0 & 0 & 4 \eta^{3} p q \\
0 & 0 & 0 & -4 \kappa^{2} \eta p q & -4 \kappa \eta^{2} p q & 0 \\
\eta p^{3} q & p^{3} q^{2} & \eta p^{3} q & \eta^{2} p^{3} & \kappa \eta p^{3} & -\kappa p^{3} q \\
\eta p^{3} q & -p^{3} q^{2} & \eta p^{3} q & -\eta^{2} p^{3} & -\kappa \eta p^{3} & -\kappa p^{3} q \\
-\eta p q^{3} & i p^{2} q^{3} & \eta p q^{3} & -i \eta^{2} q^{3} & i \kappa \eta q^{3} & -\kappa p q^{3} \\
-\eta p q^{3} & -i p^{2} q^{3} & \eta p q^{3} & i \eta^{2} q^{3} & -i \kappa \eta q^{3} & -\kappa p q^{3}
\end{array}\right)
$$

where $p=\sqrt{\eta^{2}+\kappa^{2}}$ and $q=\sqrt{\eta^{2}-\kappa^{2}}$. Then

$$
M_{j}^{\text {curve }}=P \cdot\left(\begin{array}{cccccc}
1 & l \kappa & 0 & 0 & 0 & 0 \\
0 & 1 & 0 & 0 & 0 & 0 \\
0 & 0 & e^{l \sqrt{\eta^{2}-\kappa^{2}}} & 0 & 0 & 0 \\
0 & 0 & 0 & e^{-l \sqrt{\eta^{2}-\kappa^{2}}} & 0 & 0 \\
0 & 0 & 0 & 0 & e^{i l \sqrt{\eta^{2}+\kappa^{2}}} & 0 \\
0 & 0 & 0 & 0 & 0 & e^{-i l \sqrt{\eta^{2}+\kappa^{2}}}
\end{array}\right) \cdot P^{-1}
$$

Although multiplying this out would produce a mess, we can use this with asymptotic approximations to produce a 20 by 20 matrix as in [13].

3. Asymptotic estimates. In [13], we found a way to convert the 6 by 6 matrices into 20 by 20 matrices, in such a way that the product of the large matrices is the determinant of the product of the small ones. The advantage of this conversion is that no cancellations occur as we multiply these matrices, so estimates to the second order in $\eta$ can be found by considering just the second order in each of the large matrices. 
Given a 6 by 6 matrix, there are 20 ways to choose 3 rows in it, and 20 ways of choosing 3 columns. We can consider all 400 ways of forming a 3 by 3 submatrix from the original, and take the determinants of all 400 matrices, forming a 20 by 20 matrix $N_{j}$. The $3 \times 6$ and $6 \times 3$ matrices convert into $1 \times 20$ and $20 \times 1$ matrices. Using some tensor algebra, we find that the determinant of the product of the $M_{j} \mathrm{~s}$ is equal to the product of the $N_{j} \mathrm{~s}$. Because the proof of this statement involves the exterior forms over the vector field, the 20 by 20 matrix $N_{j}$ is called the exterior matrix corresponding to $M_{j}$.

In [13] we computed $N_{j}^{\text {angle }}, N_{j}^{\text {III }}$, and $N_{j}^{\text {length }}$. To display the matrix $N_{j}^{\text {angle }}$, we let $\chi=\cos \phi$ and $\sigma=\sin \phi$. Then

$N_{j}^{\text {angle }}=$

$\left(\begin{array}{ccccccccccccccccccccc}\chi^{2} & 0 & 0 & 0 & 0 & 0 & 0 & \chi \sigma & 0 & 0 & 0 & \chi \sigma & 0 & 0 & 0 & 0 & 0 & 0 & -\sigma^{2} & 0 \\ 0 & \chi^{2} & 0 & 0 & 0 & 0 & 0 & 0 & \chi \sigma & 0 & 0 & 0 & \chi \sigma & 0 & 0 & 0 & 0 & 0 & 0 & -\sigma^{2} \\ 0 & 0 & \chi & 0 & 0 & 0 & 0 & 0 & 0 & \sigma & 0 & 0 & 0 & 0 & 0 & 0 & 0 & 0 & 0 & 0 \\ 0 & 0 & 0 & \chi & 0 & 0 & 0 & 0 & 0 & 0 & 0 & 0 & 0 & 0 & 0 & -\sigma & 0 & 0 & 0 & 0 \\ 0 & 0 & 0 & 0 & \chi & 0 & 0 & 0 & 0 & 0 & 0 & 0 & 0 & 0 & 0 & 0 & \sigma & 0 & 0 & 0 \\ 0 & 0 & 0 & 0 & 0 & 1 & 0 & 0 & 0 & 0 & 0 & 0 & 0 & 0 & 0 & 0 & 0 & 0 & 0 & 0 \\ 0 & 0 & 0 & 0 & 0 & 0 & 1 & 0 & 0 & 0 & 0 & 0 & 0 & 0 & 0 & 0 & 0 & 0 & 0 & 0 \\ -\chi \sigma & 0 & 0 & 0 & 0 & 0 & 0 & \chi^{2} & 0 & 0 & 0 & -\sigma^{2} & 0 & 0 & 0 & 0 & 0 & 0 & -\chi \sigma & 0 \\ 0 & -\chi \sigma & 0 & 0 & 0 & 0 & 0 & 0 & \chi^{2} & 0 & 0 & 0 & -\sigma^{2} & 0 & 0 & 0 & 0 & 0 & 0 & -\chi \sigma \\ 0 & 0 & -\sigma & 0 & 0 & 0 & 0 & 0 & 0 & \chi & 0 & 0 & 0 & 0 & 0 & 0 & 0 & 0 & 0 & 0 \\ 0 & 0 & 0 & 0 & 0 & 0 & 0 & 0 & 0 & 0 & \chi & 0 & 0 & 0 & 0 & 0 & 0 & -\sigma & 0 & 0 \\ -\chi \sigma & 0 & 0 & 0 & 0 & 0 & 0 & -\sigma^{2} & 0 & 0 & 0 & \chi^{2} & 0 & 0 & 0 & 0 & 0 & 0 & -\chi \sigma & 0 \\ 0 & -\chi \sigma & 0 & 0 & 0 & 0 & 0 & 0 & -\sigma^{2} & 0 & 0 & 0 & \chi^{2} & 0 & 0 & 0 & 0 & 0 & 0 & -\chi \sigma \\ 0 & 0 & 0 & 0 & 0 & 0 & 0 & 0 & 0 & 0 & 0 & 0 & 0 & 1 & 0 & 0 & 0 & 0 & 0 & 0 \\ 0 & 0 & 0 & 0 & 0 & 0 & 0 & 0 & 0 & 0 & 0 & 0 & 0 & 0 & 1 & 0 & 0 & 0 & 0 & 0 \\ 0 & 0 & 0 & \sigma & 0 & 0 & 0 & 0 & 0 & 0 & 0 & 0 & 0 & 0 & 0 & \chi & 0 & 0 & 0 & 0 \\ 0 & 0 & 0 & 0 & -\sigma & 0 & 0 & 0 & 0 & 0 & 0 & 0 & 0 & 0 & 0 & 0 & \chi & 0 & 0 & 0 \\ 0 & 0 & 0 & 0 & 0 & 0 & 0 & 0 & 0 & 0 & \sigma & 0 & 0 & 0 & 0 & 0 & 0 & \chi & 0 & 0 \\ -\sigma^{2} & 0 & 0 & 0 & 0 & 0 & 0 & \chi \sigma & 0 & 0 & 0 & \chi \sigma & 0 & 0 & 0 & 0 & 0 & 0 & \chi^{2} & 0 \\ 0 & -\sigma^{2} & 0 & 0 & 0 & 0 & 0 & 0 & \chi \sigma & 0 & 0 & 0 & \chi \sigma & 0 & 0 & 0 & 0 & 0 & 0 & \chi^{2}\end{array}\right)$

To display $N_{j}^{\mathrm{III}}$, we need to introduce some new variables. Let

$$
\begin{aligned}
t & =b e-c d, \\
r & =a d-b^{2}, \\
u & =b c-a e, \\
g & =a f-c^{2}, \\
s & =c e-b f, \\
v & =d f-e^{2}, \\
w & =a d f+2 b e c-c^{2} d-e^{2} a-b^{2} f .
\end{aligned}
$$


Then $N_{j}^{\mathrm{III}}=$

$$
\left(\begin{array}{ccccccccccccccccccccc}
\eta^{2} & 0 & 0 & 0 & 0 & 0 & 0 & 0 & 0 & 0 & 0 & 0 & 0 & i b \eta & 0 & 0 & 0 & 0 & 0 & 0 \\
i f \eta^{3} & \eta^{2} & 0 & -i c \eta^{2} & 0 & 0 & 0 & 0 & 0 & 0 & i e \eta^{2} & 0 & 0 & s \eta^{2} & i b \eta & 0 & 0 & 0 & 0 & 0 \\
i c \eta^{2} & 0 & \eta^{2} & -i a \eta & 0 & 0 & 0 & 0 & 0 & 0 & 0 & i e \eta^{2} & 0 & -u \eta & 0 & i b \eta & 0 & 0 & 0 & 0 \\
0 & 0 & 0 & \eta^{2} & 0 & 0 & 0 & 0 & 0 & 0 & 0 & 0 & 0 & i e \eta^{2} & 0 & 0 & 0 & 0 & 0 & 0 \\
i e \eta^{2} & 0 & 0 & 0 & \eta^{2} & 0 & 0 & -i c \eta^{2} & 0 & 0 & i d \eta & 0 & 0 & -t \eta & 0 & 0 & 0 & i b \eta & 0 & 0 \\
i b \eta & 0 & 0 & 0 & 0 & \eta^{2} & 0 & -i a \eta & 0 & 0 & 0 & i d \eta & 0 & r & 0 & 0 & 0 & 0 & i b \eta & 0 \\
s \eta^{2} & i b \eta & -i e \eta^{2} & u \eta & -i c \eta^{2} & i f \eta^{3} & \eta^{2} & g \eta^{2} & -i a \eta & i c \eta^{2} & -t \eta & -v \eta^{2} & i d \eta & i w \eta & r & t \eta & -i e \eta^{2} & u \eta & s \eta^{2} & i b \eta \\
0 & 0 & 0 & 0 & 0 & 0 & 0 & \eta^{2} & 0 & 0 & 0 & 0 & 0 & i d \eta & 0 & 0 & 0 & 0 & 0 & 0 \\
0 & 0 & 0 & -i e \eta^{2} & 0 & 0 & 0 & i f \eta^{3} & \eta^{2} & 0 & 0 & 0 & 0 & -v \eta^{2} & i d \eta & 0 & 0 & -i e \eta^{2} & 0 & 0 \\
0 & 0 & 0 & -i b \eta & 0 & 0 & 0 & i c \eta^{2} & 0 & \eta^{2} & 0 & 0 & 0 & i \eta & 0 & i d \eta & 0 & 0 & -i e \eta^{2} & 0 \\
0 & 0 & 0 & 0 & 0 & 0 & 0 & 0 & 0 & 0 & \eta^{2} & 0 & 0 & -i c \eta^{2} & 0 & 0 & 0 & 0 & 0 & 0 \\
0 & 0 & 0 & 0 & 0 & 0 & 0 & 0 & 0 & 0 & 0 & \eta^{2} & 0 & -i a \eta & 0 & 0 & 0 & 0 & 0 & 0 \\
0 & 0 & 0 & 0 & 0 & 0 & 0 & 0 & 0 & 0 & -i c \eta^{2} & i f \eta^{3} & \eta^{2} & g \eta^{2} & -i a \eta & i c \eta^{2} & 0 & 0 & 0 & 0 \\
0 & 0 & 0 & 0 & 0 & 0 & 0 & 0 & 0 & 0 & 0 & 0 & 0 & \eta^{2} & 0 & 0 & 0 & 0 & 0 & 0 \\
0 & 0 & 0 & 0 & 0 & 0 & 0 & 0 & 0 & 0 & 0 & 0 & 0 & i f \eta^{3} & \eta^{2} & 0 & 0 & 0 & 0 & 0 \\
0 & 0 & 0 & 0 & 0 & 0 & 0 & 0 & 0 & 0 & 0 & 0 & 0 & i c \eta^{2} & 0 & \eta^{2} & 0 & 0 & 0 & 0 \\
0 & 0 & 0 & 0 & 0 & 0 & 0 & 0 & 0 & 0 & -i b \eta & i e \eta^{2} & 0 & -u \eta & 0 & 0 & \eta^{2} & -i a \eta & i c \eta^{2} & 0 \\
0 & 0 & 0 & 0 & 0 & 0 & 0 & 0 & 0 & 0 & 0 & 0 & 0 & i e \eta^{2} & 0 & 0 & 0 & \eta^{2} & 0 & 0 \\
0 & 0 & 0 & 0 & 0 & 0 & 0 & 0 & 0 & 0 & 0 & 0 & 0 & i b \eta & 0 & 0 & 0 & 0 & \eta^{2} & 0 \\
0 & 0 & 0 & 0 & 0 & 0 & 0 & 0 & 0 & 0 & 0 & 0 & 0 & s \eta^{2} & i b \eta & -i e \eta^{2} & 0 & -i c \eta^{2} & i f \eta^{3} & \eta^{2}
\end{array}\right)
$$

Finally, the end matrices are converted to

$$
\begin{aligned}
& N_{0}^{\text {clamp }}=(0,0,0,0,0,0,0,0,0,0,0,0,0,1,0,0,0,0,0,0), \\
& N_{n}^{\text {clamp }}=(0,0,0,0,0,0,1,0,0,0,0,0,0,0,0,0,0,0,0,0)^{\mathrm{T}}, \\
& N_{n}^{\text {free }}=(0,0,0,0,0,0,0,0,0,0,0,0,0,1,0,0,0,0,0,0)^{\mathrm{T}} .
\end{aligned}
$$

Rather than displaying the exterior matrix $N_{j}^{\text {length }}$ here, we will first make some asymptotic approximations on it by assuming, without loss of generality, that the argument of $\eta$ is between 0 and $\pi / 4$. The result, after multiplying by $8 e^{l(i \eta-\eta)}$, is

$$
\widetilde{N}_{j}^{\text {length }}=\left(\begin{array}{c|c|c|c}
\widetilde{A} & 0 & 0 & 0 \\
\hline 0 & \widetilde{B} & 0 & 0 \\
\hline 0 & 0 & \widetilde{B} & 0 \\
\hline 0 & 0 & 0 & \widetilde{A}
\end{array}\right),
$$

where

$$
\tilde{A}=\left(\begin{array}{cccc}
2 x^{l} & 2 x^{l} & 2 x^{l} & 2 x^{l} \\
2 x^{l} & 2 x^{l} & 2 x^{l} & 2 x^{l} \\
2 x^{l} & 2 x^{l} & 2 x^{l} & 2 x^{l} \\
2 x^{l} & 2 x^{l} & 2 x^{l} & 2 x^{l}
\end{array}\right)
$$


and

$$
\widetilde{B}=\left(\begin{array}{cccccc}
x^{2 l}+1 & \left(\begin{array}{c}
(1-i) x^{2 l} \\
+1+i
\end{array}\right) & -i x^{2 l}+i & -i x^{2 l}+i & \left(\begin{array}{c}
(-1-i) x^{2 l} \\
-1+i
\end{array}\right) & -x^{2 l}-1 \\
\left(\begin{array}{c}
1+i) x^{2 l} \\
+1-i
\end{array}\right) & 2 x^{2 l}+2 & \left(\begin{array}{c}
(1-i) x^{2 l} \\
+1+i
\end{array}\right) & \left(\begin{array}{c}
(1-i) x^{2 l} \\
+1+i
\end{array}\right) & -2 i x^{2 l}+2 i & \left(\begin{array}{c}
(-1-i) x^{2 l} \\
-1+i
\end{array}\right) \\
i x^{2 l}-i & \left(\begin{array}{c}
(1+i) x^{2 l} \\
+1-i
\end{array}\right) & x^{2 l}+1 & x^{2 l}+1 & \left(\begin{array}{c}
(1-i) x^{2 l}+1 \\
+1+i
\end{array}\right) & -i x^{2 l}+i \\
i x^{2 l}-i & \left(\begin{array}{c}
1+i) x^{2 l} \\
+1-i
\end{array}\right) & x^{2 l}+1 & x^{2 l}+1 & \left(\begin{array}{c}
(1-i) x^{2 l} \\
+1+i
\end{array}\right) & -i x^{2 l}+i \\
\left(\begin{array}{c}
(1-i) x^{2 l} \\
-1-i
\end{array}\right) & 2 i x^{2 l}-2 i & \left(\begin{array}{c}
(1+i) x^{2 l} \\
+1-i
\end{array}\right) & \left(\begin{array}{c}
(1+i)^{2 l} \\
+i-i
\end{array}\right) & 2 x^{2 l}+2 & \left(\begin{array}{c}
(1-i) x^{2 l} \\
+1+i
\end{array}\right) \\
-x^{2 l}-1 & \left(\begin{array}{c}
(1-i) x^{2 l} \\
-1-i
\end{array}\right) & i x^{2 l}-i & i x^{2 l}-i & \left(\begin{array}{c}
(1+i) x^{2 l} \\
+1-i
\end{array}\right) & x^{2 l}+1
\end{array}\right) .
$$

Here, $x=e^{i \eta}$.

We want to also find approximations for the exterior matrix of $M_{j}^{\text {curve }}$. To ease the task of displaying the results, we will use the notation introduced in [12]. For any $m$ by $n$ matrix $A$, let $A^{\mathrm{F}}$ be the $m$ by $n$ matrix formed by "flipping" $A$ over, i.e., for an $m$ by $n$ matrix,

$$
\left(A^{\mathrm{F}}\right)_{i j}=(A)_{(m-i+1),(n-j+1)} .
$$

For example,

$$
\left(\begin{array}{cc}
3 & -4 \\
6 & -1 \\
-2 & 5
\end{array}\right)^{\mathrm{F}}=\left(\begin{array}{cc}
5 & -2 \\
-1 & 6 \\
-4 & 3
\end{array}\right)
$$

This differs from the standard transpose, denoted $A^{\mathrm{T}}$. Using MATHEMATICA, we find that, to second order in $\eta$,

$$
\widetilde{N}_{j}^{\text {curve }}=\left(\begin{array}{c|c|c|c}
\eta \tilde{A} & -\kappa \widetilde{F}^{\mathrm{FT}} & -\kappa \widetilde{D}^{\mathrm{FT}} & 0 \\
\hline \kappa \widetilde{C} & \eta \widetilde{B} & 0 & \kappa \widetilde{D} \\
\hline \kappa \widetilde{E} & -\kappa^{2} l \widetilde{B} & \eta \widetilde{B} & \kappa \widetilde{F} \\
\hline 0 & -\kappa \widetilde{E}^{\mathrm{FT}} & -\kappa \widetilde{C}^{\mathrm{FT}} & \eta \widetilde{A}
\end{array}\right),
$$

where $\tilde{A}$ and $\widetilde{B}$ were given above,

$$
\begin{gathered}
\tilde{C}=\left(\begin{array}{cccc}
-2 x^{l} & 1-2 x^{l}+x^{2 l} & 1+i-2 x^{l}+(1-i) x^{2 l} & i-2 x^{l}-i x^{2 l} \\
-2 x^{l} & 1-i-2 x^{l}+(1+i) x^{2 l} & 2-2 x^{l}+2 x^{2 l} & 1+i-2 x^{l}+(1-i) x^{2 l} \\
0 & -i+i x^{2 l} & (1-i)+(1+i) x^{2 l} & 1+x^{2 l} \\
-2 x^{l} & -i-2 x^{l}+i x^{2 l} & 1-i-2 x^{l}+(1+i) x^{2 l} & 1-2 x^{l}+x^{2 l} \\
0 & (-1-i)+(-1+i) x^{2 l} & -2 i+2 i x^{2 l} & (1-i)+(1+i) x^{2 l} \\
0 & -1-x^{2 l} & (-1-i)+(-1+i) x^{2 l} & -i+i x^{2 l}
\end{array}\right), \\
\widetilde{D}=\left(\begin{array}{cccc}
(1+i)+(1-i) x^{2 l} & i-i x^{2 l} & 0 & 1+x^{2 l} \\
2-2 x^{l}+2 x^{2 l} & 1+i-2 x^{l}+(1-i) x^{2 l} & -2 x^{l} & 1-i-2 x^{l}+(1+i) x^{2 l} \\
(1-i)+(1+i) x^{2 l} & 1+x^{2 l} & 0 & -i+i x^{2 l} \\
1-i-2 x^{l}+(1+i) x^{2 l} & 1-2 x^{l}+x^{2 l} & -2 x^{l} & -i-2 x^{l}+i x^{2 l} \\
-2 i+2 i x^{2 l} & (1-i)+(1+i) x^{2 l} & 0 & (-1-i)+(-1+i) x^{2 l} \\
-1-i+2 x^{l}+(-1+i) x^{2 l} & -i+2 x^{l}+i x^{2 l} & 2 x^{l} & -1+2 x^{l}-x^{2 l}
\end{array}\right),
\end{gathered}
$$




$$
\begin{aligned}
& \widetilde{E}=\left(\begin{array}{cccc}
-i+i x^{2 l} & (1-i)+(1+i) x^{2 l} & 1+x^{2 l} & 0 \\
(-1-i)+(-1+i) x^{2 l} & -2 i+2 i x^{2 l} & (1-i)+(1+i) x^{2 l} & 0 \\
-1-x^{2 l} & (-1-i)+(-1+i) x^{2 l} & -i+i x^{2 l} & 0 \\
-1+2 x^{l}-x^{2 l} & -1-i+2 x^{l}+(i-1) x^{2 l} & -i+2 x^{l}+i x^{2 l} & 2 x^{l} \\
i-1+2 x^{l}+(-1-i) x^{2 l} & -2+2 x^{l}-2 x^{2 l} & -1-i+2 x^{l}+(i-1) x^{2 l} & 2 x^{l} \\
i+2 x^{l}-i x^{2 l} & i-1+2 x^{l}+(-1-i) x^{2 l} & -1+2 x^{l}-2 x^{2 l} & 2 x^{l}
\end{array}\right), \\
& \widetilde{F}=\left(\begin{array}{cccc}
1-2 x^{l}+x^{2 l} & -2 x^{l} & -i-2 x^{l}+i x^{2 l} & 1-i-2 x^{l}+(1+i) x^{2 l} \\
(1-i)+(1+i) x^{2 l} & 0 & (-1-i)+(-1+i) x^{2 l} & -2 i+2 i x^{2 l} \\
-i+i x^{2 l} & 0 & -1-x^{2 l} & (-1-i)+(-1+i) x^{2 l} \\
-i+2 x^{l}+i x^{2 l} & 2 x^{l} & -1+2 x^{l}-x^{2 l} & -1-i+2 x^{l}+(i-1) x^{2 l} \\
-1-i+2 x^{l}+(i-1) x^{2 l} & 2 x^{l} & i-1+2 x^{l}+(-1-i) x^{2 l} & -2+2 x^{l}-2 x^{2 l} \\
-1-x^{2 l} & 0 & i-i x^{2 l} & (-1+i)+(-1-i) x^{2 l}
\end{array}\right) .
\end{aligned}
$$

Notice that if the curvature $\kappa=0$, then this reduces to $\eta N_{j}^{\text {length }}$ for a straight beam.

At this point, all eigenfrequencies can be approximated by finding the roots to the equation

$$
\tilde{N}_{0} \cdot \tilde{N}_{1} \cdot \tilde{N}_{2} \cdots \tilde{N}_{n}=0
$$

To compute the positions of the streams of the eigenvalues we can use the results from [13]. If the lengths are all integers, we can divide (3.1) by factors of $\eta$ to obtain an equation of the form

$$
f(x)+\frac{g(x)}{\eta}=O\left(\eta^{-2}\right)
$$

for some polynomial functions $f(x)$ and $g(x)$. If we let $r$ be a root of $f(x)=0$, then there will be two possibilities. If $|r|=1$, then

$$
\lambda_{k} \sim \frac{-2 g(r)}{f^{\prime}(r) r}+(2 k \pi+\arg (r))^{2} i .
$$

If $|r| \neq 1$, then

$$
\lambda_{k} \sim 2 \log |r|(2 k \pi+\arg (r))-\frac{2 g(r)}{f^{\prime}(r) r}+\left((2 k \pi+\arg (r))^{2}-2 \log ^{2}|r|\right) i .
$$

In [14], it was proved that all streams were in fact linear. The basis for this proof involved observing patterns in the matrices $\widetilde{N}_{j}$. In particular, we had that

$$
\bar{x}^{2 l} \widetilde{N}_{j}^{\text {length }}(1 / \bar{x})=\overline{\widetilde{N}_{j}^{\text {length }}(x)} .
$$

Notice that the same pattern occurs in the $\widetilde{N}_{j}^{\text {curve }}$, that is,

$$
\bar{x}^{2 l} \widetilde{N}_{j}^{\text {curve }}(1 / \bar{x})=\overline{\widetilde{N}_{j}^{\text {curve }}(x)} .
$$

Thus, by the same reasoning given in [14], the streams of eigenfrequencies will all be linear, even if the beams are curved. 
As an example, we can use the large matrices to find the approximate eigenfrequencies of the structures in Figure 1. For simplicity, we will take $m E I=1$. In this case, the damper is a type III, with $a=K$ and $b=c=d=e=f=0$. After multiplying the matrices together, and dividing by a factor of $\eta^{3}$, we obtain (3.2), with

$$
f(x)=-8 \pi\left(1+x^{2 \pi}\right)
$$

and

$$
\begin{aligned}
g(x)= & -4-4 i+(2+2 i) K \pi+(16+16 i) x^{\pi / 2}-40 x^{\pi}+4 i K \pi x^{\pi} \\
& +(16-16 i) x^{3 \pi / 2}+(-4+4 i) x^{2 \pi}+(-2+2 i) K \pi x^{2 \pi} .
\end{aligned}
$$

Since the lengths of the beams were not rational, $f(x)$ and $g(x)$ are not polynomials in $x$. However, we can change variables to make them polynomials. If we let $\eta^{\prime}=\pi \eta / 2$ and $x^{\prime}=e^{i \eta^{\prime}}=x^{\pi / 2}$, then (3.2) becomes

$$
f\left(x^{\prime}\right)+\frac{g\left(x^{\prime}\right)}{\eta^{\prime}}=O\left(\eta^{\prime-2}\right)
$$

with

$$
f\left(x^{\prime}\right)=-8 \pi\left(1+x^{\prime 4}\right)
$$

and

$$
\begin{aligned}
g\left(x^{\prime}\right)= & (-2-2 i) \pi+(1+i) K \pi^{2}+(8+8 i) \pi x^{\prime}-20 \pi x^{\prime 2}+2 i K \pi^{2} x^{\prime 2} \\
& +(8-8 i) \pi x^{\prime 3}+(-2+2 i) \pi x^{\prime 4}+(-1+i) K \pi^{2} x^{\prime 4} .
\end{aligned}
$$

We can apply (3.3) to obtain an estimate for $i \eta^{\prime 2}$. We obtain the four linear streams

$$
\begin{aligned}
& i n_{1, k}^{\prime 2} \sim\left((2 \pi k+\pi / 4)^{2}+3 / 2-\sqrt{2}-K \pi / 8\right) i, \\
& i n_{2, k}^{\prime 2} \sim-K \pi / 4+\left((2 \pi k+3 \pi / 4)^{2}-1-K \pi / 8\right) i, \\
& i n_{3, k}^{\prime 2} \sim\left((2 \pi k+5 \pi / 4)^{2}+3 / 2+\sqrt{2}-K \pi / 8\right) i, \\
& i n_{4, k}^{\prime 2} \sim-K \pi / 4+\left((2 \pi k+7 \pi / 4)^{2}-1-K \pi / 8\right) i .
\end{aligned}
$$

Since $\lambda=i \eta^{2}=4 i \eta^{\prime} / \pi^{2}$, we have

$$
\begin{aligned}
& \lambda_{1, k} \sim\left((4 k+1 / 2)^{2}+6 / \pi^{2}-4 \sqrt{2} / \pi^{2}-K / 2 \pi\right) i, \\
& \lambda_{2, k} \sim-K / \pi+\left((4 k+3 / 2)^{2}-4 / \pi^{2}-K / 2 \pi\right) i, \\
& \lambda_{3, k} \sim\left((4 k+5 / 2)^{2}+6 / \pi^{2}+4 \sqrt{2} / \pi^{2}-K / 2 \pi\right) i, \\
& \lambda_{4, k} \sim-K / \pi+\left((4 k+7 / 2)^{2}-4 / \pi^{2}-K / 2 \pi\right) i .
\end{aligned}
$$

Much of the work in this paper required the use of the symbolic manipulator MATHEMATICA running on a SUN Microsystems workstation for the computation of the large matrices.

Although this paper analyzes a linear model of a physical system, the experimental data cited in [5] and [6] indicate that this model is an accurate one. However, we still are only considering vibrations that occur within the plane. Hopefully, analysis for structures that do not lie in a plane can be done using a similar technique, and this will be treated in a future work. 


\section{REFERENCES}

[1] C. Aganovic and Z. Tutez, A justification of the one-dimensional model of an elastic beam, Math. Methods Appl. Sci. 8, 1-14 (1986)

[2] W. Boothby, An Introduction to Differentiable Manifolds and Riemannian Geometry, Academic Press, Orlando, FL, 1986

[3] G. Chen, M. C. Delfour, A. M. Krall, and G. Payne, Modeling, stabilization, and control of serially connected beams, SIAM J. Control Optim. 25, 526-546 (1987)

[4] G. Chen, S. G. Krantz, D. W. Ma, C. E. Wayne, and H. H. West, The Euler-Bernoulli beam equation with boundary energy dissipation, Operator Methods for Optimal Control Problems, Marcel Dekker, New York, 1987, pp. 67-96

[5] G. Chen, S. G. Krantz, D. L. Russell, C. E. Wayne, H. H. West, and M. P. Colman, Analysis, designs and behavior of dissipative joints for coupled beams, SIAM J. Appl. Math. 49, 1665-1693 (1989)

[6] G. Chen, S. G. Krantz, D. L. Russell, C. E. Wayne, H. H. West, and J. Zhou, Modeling, analysis and testing of dissipative beam joints-experiments and data smoothing, Math. Comput. Modelling 11, 1011-1016 (1988)

[7] G. Chen and H. Wang, Asymptotic locations of eigenfrequencies of Euler-Bernoulli beam with nonhomogeneous structural and viscous damping coefficients, SIAM J. Control Optim. 29, 347-367 (1991)

[8] G. Chen and J. Zhou, The wave propagation method for the analysis of boundary stabilization in vibration structures, SIAM J. Appl. Math. 50, 1254-1283 (1990)

[9] P. G. Ciarlet, Plates and Junctions in Elastic Multi-structures, Springer-Verlag, New York, 1990

[10] J. B. Keller and S. I. Rubinow, Asymptotic solution of eigenvalue problems, Ann. of Physics 9, 24-75 (1960)

[11] A. M. Krall, Asymptotic stability of the Euler-Bernoulli beam with boundary control, J. Math. Anal. Appl. 137, 288-295 (1989)

[12] S. G. Krantz and W. Paulsen, Asymptotic eigenfrequency distributions for the $N$-beam EulerBernoulli coupled beam equation with dissipative joints, J. Symbolic Comput. 11, 369-418 (1991)

[13] W. H. Paulsen, Eigenfrequencies of non-collinearly coupled beams with dissipative joints, Proc. 31 st IEEE Conf. Decision and Control (Tucson, AZ, 1992), Vol. 3, IEEE Control Systems Soc., New York, 1992, pp. 2986-2991

[14] W. H. Paulsen, Eigenfrequencies of the non-collinearly coupled Euler-Bernoulli beam system with dissipative joints, preprint

[15] W. D. Pilkey, Manual for the response of structural members. Vol. I, Illinois Inst. Tech. Res. Inst. Project J6094, Chicago, IL, 1969 